\title{
Improving Health Equity and Reducing Disparities in Pediatric and Adolescent/Young Adult Oncology: In Support of Clinical Practice Guidelines
}

\author{
Justine M. Kahn, MD, MS, ${ }^{1}$ and Melissa Beauchemin, PhD, RN, CPNP ${ }^{1}$
}

\begin{abstract}
Despite extraordinary strides in cancer therapy over the past 30 years, racial/ethnic, socioeconomic, and age-related survival disparities persist. Hodgkin lymphoma offers an excellent paradigm to understand these disparities because successful approaches are well established in both the up-front and relapsed treatment settings. The following review, which accompanies the 2021 NCCN Guidelines for Pediatric Hodgkin Lymphoma, suggests that systemic inequities in cancer care disproportionately affect minority and low-income children, adolescents, and young adults, and directly contribute to observed disparities in cancer-related outcomes. It proposes that the first step toward reducing disparities is large-scale dissemination of guidelines, because equity is best achieved when treatment approaches are clear, comprehensive, and standardized across all clinical practice settings.
\end{abstract}

J Natl Compr Canc Netw 2021;19(6):765-769 doi: 10.6004/jnccn.2021.7048

${ }^{1}$ Division of Pediatric Hematology/Oncology/Stem Cell Transplantation, Herbert Irving Comprehensive Cancer Center, Columbia University Irving Medical Center, New York, NY.
"Of all the forms of inequality, injustice in health is the most shocking and inhuman."

- Martin Luther King Jr.

Despite extraordinary strides in cancer therapy over the past 3 decades, racial/ethnic and socioeconomic disparities persist. ${ }^{1-3}$ Hodgkin lymphoma (HL) represents an ideal paradigm for understanding cancer disparities, because successful treatments are well established in both the up-front and relapse treatment settings. Today, 5year overall survival (OS) rates for children, adolescents, and young adults (AYA; aged 15-39 years) with HL exceed $95 \%$, with event-free survival (EFS) rates $>85 \%{ }^{4}$ The treatment successes in HL are attributed, in part, to 4 key factors: (1) research to identify disease-related predictors of poor outcome; (2) informed use of risk-based, response-adapted, combined-modality therapy,6; (3) improvements in supportive care and symptom management; and (4) refinements in therapy to reduce the risk of long-term treatment-related morbidities. ${ }^{7-9}$ Accordingly, it is important to examine why some populations have not benefitted from these advances to focus efforts on improving outcomes for all patients. ${ }^{1,10-12}$

In 2011, the Institute of Medicine defined clinical practice guidelines as "statements that include recommendations intended to optimize patient care that are informed by a systematic review of evidence and an assessment of the benefits and harms of alternative care options."13 This review, which accompanies the 2021 NCCN Clinical Practice Guidelines in Oncology (NCCN Guidelines) for Pediatric HL elsewhere in this issue, focuses on the vulnerable populations who continue to trail behind on survival curves. Its premise is that systemic inequities in care over the cancer continuum disproportionately affect minority and low-income patients and directly contribute to observed disparities in cancerrelated outcomes. This review highlights where inequities may occur and suggests that improving health equity requires wide dissemination and uptake of guidelines to ensure best care for all patients. Finally, it argues that 
vulnerable populations in the United States may serve to benefit most from national clinical practice guidelines, as equity is best achieved when treatment approaches are clear, comprehensive, and standardized across all clinical practice settings.

\section{Defining Disparities, Inequities, and Social Determinants of Health}

The WHO defines disparities as avoidable differences in health arising from both the social and economic conditions that determine an individual's risk of illness and the actions taken to prevent or treat said illness. ${ }^{14}$ The groups who systematically experience worse health outcomes are known as vulnerable populations. Inequities are differences in the distribution of health resources between population groups that arise from the social conditions in which people are born, grow, live, work, and age. These social conditions, also known as social determinants of health $(\mathrm{SDOH})$, broadly can be categorized as economic, education, social and community context, neighborhood, and the built environment. They include upstream factors such as political, socioeconomic, and cultural constructs, as well as place-based factors such as access to healthcare, transportation, education systems, safe neighborhoods, and availability of healthy foods. In combination with individual characteristics, $\mathrm{SDOH}$ are important drivers of racial disparities in cancer-related outcomes. ${ }^{15}$

Nationally representative studies of children and AYAs with HL indicate that non-Hispanic (NH) Black and Hispanic race/ethnicity are associated with worse survival. ${ }^{1,11,12}$ One pathway hypothesized to be an important link between race/ethnicity and cancer outcomes are the social and economic factors impacting access to care. ${ }^{16}$ Given that Black and Hispanic children in the United States have disproportionate social, economic, and environmental disadvantages, the influence of $\mathrm{SDOH}$ on cancer care and survival cannot be overlooked..$^{17,18}$

\section{The Cancer Continuum}

The cancer continuum illustrates the course of cancer from prediagnosis, through treatment, into survivorship, end of life, and death. ${ }^{19}$ It can be argued that the effects of social determinants on pediatric and AYA cancer outcomes (Figure 1A) are mediated through multilevel factors impacting healthcare access and cancer care over the full cancer continuum (Figure 1B, C). We propose that clinical practice guidelines are key to mitigating the variations in care that result from social disadvantage at each level.

\section{Pretreatment Phase}

Along the cancer continuum, the pretreatment phase encompasses the patient's baseline sociodemographic characteristics, as well as access-related factors impacting prediagnosis care, including screening (for some cancers), diagnostic workup, referrals, and time to initiation of therapy. Apart from patients with inherited cancer predisposition syndromes, and in contrast to many adult-onset malignancies (eg, colon, breast, prostate), screening for childhood cancers is uncommon. Diagnostic workup, referral, and expeditious initiation of therapy are therefore critical. Despite guidelines for pretreatment evaluation, staging, and treatment planning, however, disparities in both the completeness and timeliness of workup have been reported, specifically in AYAs with public insurance. ${ }^{20}$ Even before workup, studies demonstrate that patients who are non-White, economically disadvantaged, or publicly insured frequently present with advanced-stage disease. ${ }^{21-24}$ In a registry analysis of 58,000 AYAs, having public or no insurance was associated with progressively higher odds of late stage at presentation for nearly all cancer sites. ${ }^{25}$ In analyses of patients with HL, there was a 2fold increased risk of death due to advanced stage. ${ }^{26}$ In HL, advanced stage necessitates more intensive therapy, which in turn is associated with increased risk of both short- and long-term treatment-related toxicities.

\section{During Therapy}

Discrete components of care after diagnosis, including treatment location, ${ }^{27}$ supportive care, and clinical trial enrollment, are associated with optimal outcomes in HL.

\section{Treatment}

Using data from the California Cancer Registry, Keegan et $\mathrm{al}^{25}$ previously reported a significant difference in likelihood of being treated at an NCI or Children's Oncology Group (COG) affiliate among patients with HL who have public versus private insurance. ${ }^{28}$ This, in turn, was associated with higher hazard of death in the publicly insured group. Although more research is needed to understand exactly how location of care impacts survival, proposed hypotheses for superior outcomes at NCI/ COG facilities may include higher clinical trial enrollment rates and more access to tertiary-level supportive and postrelapse care, including hematopoietic cell transplantation and novel salvage regimens. Along these lines, in HL, use of combined-modality therapy (chemotherapy and radiation therapy) has led to substantial improvements in survival over time, particularly in children. Numerous studies have shown that Black patients are less likely than White patients to receive radiation therapy during treatment for HL, and that this, in turn, impacts outcomes. ${ }^{24,29,30}$

\section{Clinical Trial Enrollment}

These care differences are largely mitigated by enrollment on clinical trials. ${ }^{4-6,31}$ We recently reported that $\mathrm{NH}$ White, NH Black, and Hispanic patients enrolled on 

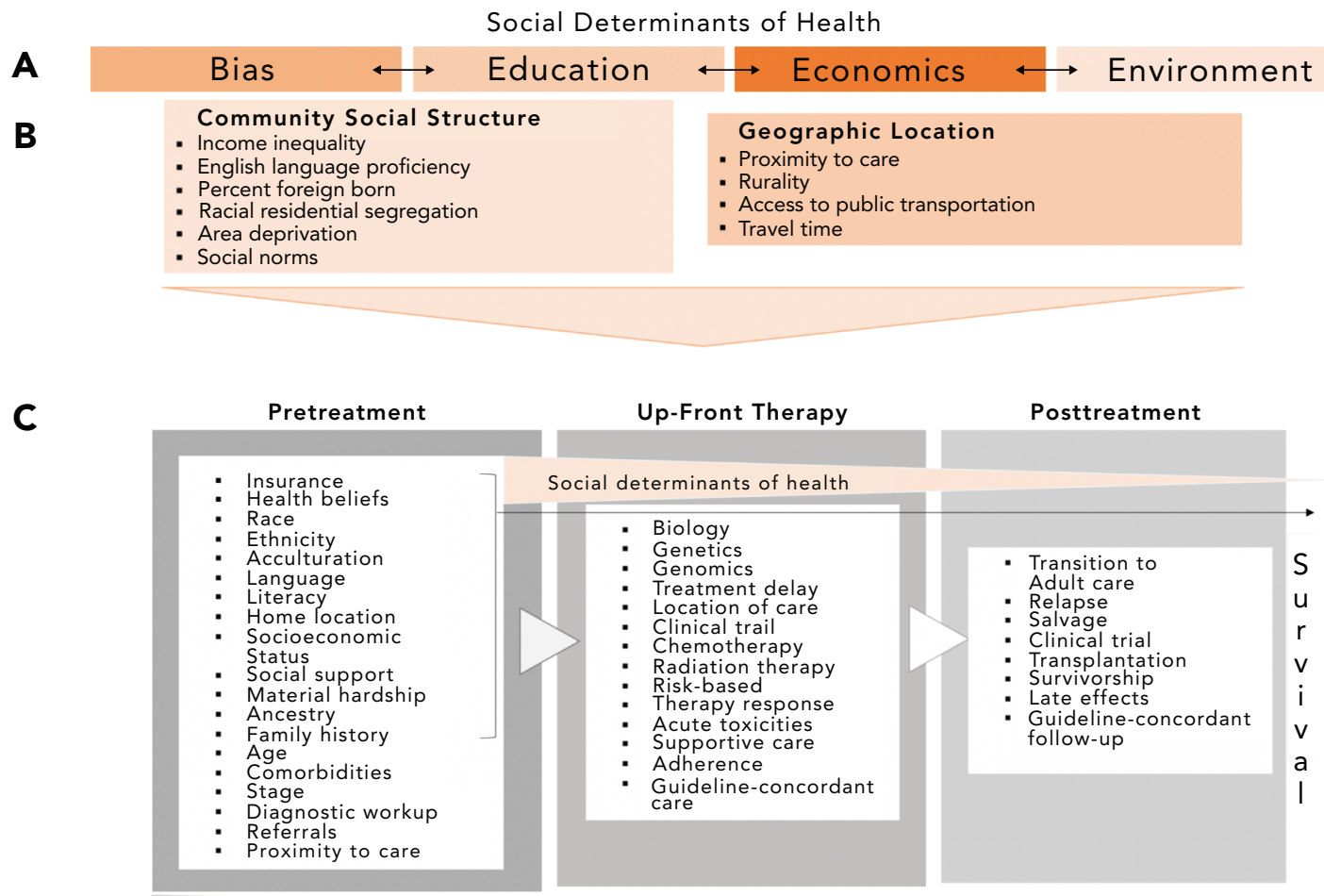

Cancer continuum

Figure 1. (A) Societal, (B) community, and (C) individual levels of influence on cancer care and outcomes. These levels shape how a person interacts with the healthcare system over the cancer continuum from prediagnosis through therapy, and into posttreatment follow-up.

phase III COG trials for treatment of newly diagnosed HL had comparable relapse rates and similar EFS (relapse, progression, second malignant neoplasm, death). This finding indicated that treatment with dose-dense, response-adapted therapy for newly diagnosed HL reduced previously reported racial/ethnic differences in EFS.

\section{Salvage Therapy}

In the previously mentioned patients treated on COG studies for newly diagnosed HL, survival disparities by race/ethnicity were observed among those with relapsed disease. According to protocol design, patients with disease recurrence were removed from trials and received salvage therapy per the treating team's discretion. In this subgroup of patients with recurrent disease, postrelapse survival probabilities were $67 \%$ in NH Black patients, $80 \%$ in Hispanic patients, and $87 \%$ in $\mathrm{NH}$ White patients $(P<.01) .^{15}$ In multivariable models, Hispanic patients had 2.7-fold increased risk of death $(95 \%$ CI, 1.2-6.2), and NH Black patients had 3.5-fold increased risk of death (95\% CI, 1.5-8.0) compared with the NH White group. ${ }^{15}$ This striking difference in risk of death by race/ethnicity raises the possibility that differences in salvage therapy or supportive care outside of the cooperative group setting contributed to postrelapse disparities. ${ }^{1,49}$ Given that HL is a diagnosis of adolescents primarily, it is possible that after relapse, patients aged out of Pediatrics and transitioned to Medical Oncology for salvage care. This transition to Medical Oncology may impact outcomes, particularly in lowincome or other vulnerable populations, but additional research is needed to explore this further.

\section{Supportive Care}

Evidence-based supportive care guidelines are well established, and have been shown to improve tolerability of treatment, adherence, and health-related quality of life in patients with cancer. ${ }^{32-34}$ Despite the known benefit of supportive care guidelines, studies demonstrate that they are followed inconsistently, particularly in minority and low-income populations. ${ }^{31,35}$ This is well-documented with chemotherapy-induced nausea and vomiting (CINV), wherein racial and socioeconomic disparities in receipt of guideline concordant care (GCC) are observed in both pediatric and medical oncology. ${ }^{36,37}$ In an analysis of GCC among children and AYAs receiving moderately and highly emetogenic chemotherapy, patients with commercial (vs public) insurance were significantly more likely to receive GCC (adjusted odds ratio, 2.4; 95\% CI, 1.1-5.2). ${ }^{38}$ The benefits of guideline-concordant supportive care, 
include improving short- and long-term adverse effects of therapy. Standardized provision of these guidelines is an important part of providing equitable care during cancer therapy, as improving health-related quality of life for patients with cancer is critical.

\section{Survivorship and Long-Term Follow-Up}

Improving the quality of long-term follow-up in children and AYAs with HL is essential to reducing cancer disparities. Inequities observed during up-front cancer therapy extend into long-term follow-up and survivorship care. Numerous studies have reported disparities in children and AYAs in the provision of equitable survivorship care for vulnerable popualtions. ${ }^{39,40}$ Studies report that minority and low-income populations are at high-risk for not receiving guideline-recommended survivorship care, which results in disparities in long-term morbidity and mortality. ${ }^{41}$ Late effects of HL therapy broadly include endocrine, pulmonary, and cardiac dysfunction, as well as risk of subsequent neoplasms. ${ }^{42}$ These risks persist decades after therapy completion, and thus inequities in access to or provision of guideline-concordant survivorship care serve to maintain the disparities that begin before diagnosis. ${ }^{21,40}$

\section{Conclusions}

It behooves us to consider why treatment location and clinical trial enrollment, for example, are consistently associated with superior outcomes in pediatric and AYA oncology. Similar to cooperative group clinical trials, centers affiliated with the NCI and COG largely follow standardized treatment and supportive care guidelines, thus reducing variation across populations. Although most children in the United States receive cancer care at NCI or COG-affiliate centers, not all enroll on trials, and underenrollment is common, particularly among younger Black patients with hematologic malignancies and those with relapsed HL. ${ }^{4,43}$ Furthermore, it is well established that AYAs are historically underenrolled on clinical trials, ${ }^{44}$ which may be related to their being more likely to receive cancer care in the community oncology setting. ${ }^{27}$ We propose that wide distribution of the new NCCN Guidelines for Pediatric HL thus has the opportunity to increase provision of GCC to vulnerable populations who may not have access to cancer centers or who may not enroll on clinical trials. With this, we propose that a critical part of establishing these new guidelines will be measuring their impact on cancer care delivery and HL outcomes in years to come, particularly among patients treated in the community oncology setting. Thus, studies should be developed to measure barriers and facilitators to guideline uptake across the United States, and the impact of this uptake on HL outcomes.

Practically speaking, eliminating cancer outcome disparities means reducing care inequities among the disadvantaged. Given the pervasiveness of healthcare inequities in the United States, establishing new clinical guidelines will not, in isolation, be enough to eliminate disparities. Multilevel interventions at the patient, provider, health systems, and societal levels will be required to improve health equity and reduce disparities in cancer medicine; however, establishing clinical practice guidelines may be a first step toward that goal. As efforts are made to expand clinical trial enrollment across underserved and vulnerable populations, similar efforts to expand the use of COG and NCCN guidelines for treatment, supportive care, and survivorship are essential.

Submitted January 18, 2021; revision received March 30, 2021; accepted for publication April 20, 2021

Disclosures: The authors have disclosed that they have not received any financial consideration from any person or organization to support the preparation, analysis, results, or discussion of this article.

Funding: This work is supported in part by the Lymphoma Research Foundation (J.M. Kahn) and by an NCl Training Program in Cancer-Related Population Sciences (NCI T32CA094061; M. Beauchemin).

Correspondence: Justine M. Kahn, MD, MS, Division of Pediatric Hematology/Oncology/Stem Cell Transplantation, Herbert Irving Comprehensive Cancer Center, Columbia University Irving Medical Center, 161 Fort Washington Avenue, IP7, New York, NY 10032.

Email: jk2034@cumc.columbia.edu

\section{References}

1. Kahn JM, Keegan TH, Tao L, et al. Racial disparities in the survival of American children, adolescents, and young adults with acute lymphoblastic leukemia, acute myelogenous leukemia, and Hodgkin lymphoma. Cancer 2016;122:2723-2730.

2. Surveillance, Epidemiology, and End Results (SEER) Program (www.seer cancer.gov) SEER*Stat Database: Incidence - SEER Research Data, 18 Registries, Nov 2019 Sub (2000-2017) - Linked To County Attributes Time Dependent (1990-2017) Income/Rurality, 1969-2018 Counties, National Cancer Institute, DCCPS, Surveillance Research Program, released April 2020, based on the November 2019 submission.

3. World Health Organization. Social determinants of health: key concepts. Accessed April 20, 2021. Available at: https://www.who.int/news-room/q a-detail/social-determinants-of-health-key-concepts

4. Kahn JM, Kelly KM, Pei Q, et al. Survival by race and ethnicity in pediatric and adolescent patients with Hodgkin lymphoma: a Children's Oncology Group study. J Clin Oncol 2019;37:3009-3017.
5. Friedman DL, Chen L, Wolden S, et al. Dose-intensive response-based chemotherapy and radiation therapy for children and adolescents with newly diagnosed intermediate-risk Hodgkin lymphoma: a report from the Children's Oncology Group Study AHOD0031. J Clin Oncol 2014;32:3651-3658

6. Keller FG, Castellino SM, Chen L, et al. Results of the AHOD0431 trial of response adapted therapy and a salvage strategy for limited stage, classical Hodgkin lymphoma: a report from the Children's Oncology Group. Cancer 2018;124:3210-3219.

7. Diefenbach CS, Connors JM, Friedberg JW, et al. Hodgkin lymphoma: current status and clinical trial recommendations. J Natl Cancer Inst 2016;109:djw249.

8. Kelly KM. Hodgkin lymphoma in children and adolescents: improving the therapeutic index. Hematology (Am Soc Hematol Educ Program) 2015;2015:514-521 
9. Kahn JM, Kelly KM. Adolescent and young adult Hodgkin lymphoma: raising the bar through collaborative science and multidisciplinary care. Pediatr Blood Cancer 2018;65:e27033.

10. Keegan TH, Ries LA, Barr RD, et al. Comparison of cancer survival trends in the United States of adolescents and young adults with those in children and older adults. Cancer 2016;122:1009-1016.

11. Evens AM, Antillon M, Aschebrook-Kilfoy B, Chiu BCH. Racial disparities in Hodgkin's lymphoma: a comprehensive population-based analysis. Ann Oncol 2012;23:2128-2137.

12. Keegan TH, Clarke CA, Chang ET, et al. Disparities in survival after Hodgkin lymphoma: a population-based study. Cancer Causes Control 2009;20:1881-1892

13. Institute of Medicine (US) Committee on Standards for Developing Trustworthy Clinical Practice Guidelines; Graham R, Mancher M, Miller Wolman D, et al, eds. Clinical Practice Guidelines We Can Trust. Washington, DC: National Academies Press; 2011

14. Braveman P. What are health disparities and health equity? We need to be clear. Public Health Rep 2014;129(Suppl 2):5-8.

15. Koh HK, Piotrowski JJ, Kumanyika S, et al. Healthy people: a 2020 vision for the social determinants approach. Health Educ Behav 2011;38:551-557.

16. Hiatt RA, Breen N. The social determinants of cancer: a challenge for transdisciplinary science. Am J Prev Med 2008;35(2 Suppl):S141-150.

17. Venkatapuram S, Marmot M. Epidemiology and social justice in light of social determinants of health research. Bioethics 2009;23:79-89.

18. Marmot MG, Bell R. Action on health disparities in the United States: commission on social determinants of health. JAMA 2009;301:1169-1171.

19. Shavers VL, Fagan $P, M c D o n a l d ~ P$. Health disparities across the cancer continuum. J Health Care Poor Underserved 2007;18(4 Suppl):1-5.

20. Parsons HM, Harlan LC, Seibel NL, et al. Clinical trial participation and time to treatment among adolescents and young adults with cancer: does age at diagnosis or insurance make a difference? J Clin Oncol 2011;29:4045-4053

21. Parsons HM, Maguire FB, Morris CR, et al. Impact of insurance type and timing of Medicaid enrollment on survival among adolescents and young adults with cancer. Pediatr Blood Cancer 2020;67:e28498.

22. Shariff-Marco S, Yang J, John EM, et al. Intersection of race/ethnicity and socioeconomic status in mortality after breast cancer. J Community Health 2015;40:1287-1299.

23. Aristizabal P. Diverse populations and enrollment in pediatric cancer clinical trials: challenges and opportunities. Pediatr Blood Cancer 2020;67:e28296.

24. Aristizabal P, Fuller S, Rivera R, et al. Improving pediatric cancer care disparities across the United States-Mexico border: lessons learned from a transcultural partnership between San Diego and Tijuana. Front Public Health 2015;3:159.

25. Keegan TH, DeRouen MC, Parsons HM, et al. Impact of treatment and insurance on socioeconomic disparities in survival after adolescent and young adult Hodgkin lymphoma: a population-based study. Cancer Epidemiol Biomarkers Prev 2016;25:264-273.

26. Rosenberg $A R$, Kroon $L, C$ hen $L$, et al. Insurance status and risk of cancer mortality among adolescents and young adults. Cancer 2015;121:1279-1286.
27. Wolfson J, Sun CL, Wyatt L, et al. Impact of treatment site on disparities in outcome among adolescent and young adults with Hodgkin lymphoma. Leukemia 2017;31:1450-1453.

28. Hoppe RT, Advani RH, Ai WZ, et al. Hodgkin lymphoma, version 2.2020, NCCN Clinical Practice Guidelines in Oncology. J Natl Compr Canc Netw 2020;18:755-781.

29. Grubb WR, Neboori HJ, Diaz AD, et al. Racial and ethnic disparities in the pediatric Hodgkin lymphoma population. Pediatr Blood Cancer 2016;63:428-435.

30. Wu J, Huang Y, Tergas Al, et al. The effect of guideline-concordant care in mitigating insurance status disparities in cervical cancer. Gynecol Oncol 2020;159:309-316.

31. Kelly KM, Sposto R, Hutchinson R, et al. BEACOPP chemotherapy is a highly effective regimen in children and adolescents with high-risk Hodgkin lymphoma: a report from the Children's Oncology Group. Blood 2011;117:2596-2603.

32. Wright JD, Neugut Al, Ananth CV, et al. Deviations from guideline-based therapy for febrile neutropenia in cancer patients and their effect on outcomes. JAMA Intern Med 2013;173:559-568.

33. Aapro M, Molassiotis A, Dicato $M$, et al. The effect of guidelineconsistent antiemetic therapy on chemotherapy-induced nausea and vomiting (CINV): the Pan European Emesis Registry (PEER). Ann Oncol 2012;23:1986-1992.

34. Olver I. The MASCC Textbook of Cancer Supportive Care and Survivorship, 2nd ed. New York, NY: Springer; 2018.

35. Patel P, Robinson PD, Orsey A, et al. Chemotherapy-induced nausea and vomiting prophylaxis: practice within the Children's Oncology Group. Pediatr Blood Cancer 2016;63:887-892.

36. Check DK, Reeder-Hayes KE, Zullig LL, et al. Examining racial variation in antiemetic use and post-chemotherapy health care utilization for nausea and vomiting among breast cancer patients. Support Care Cancer 2016;24:4839-4847.

37. Gomez DR, Liao K-P, Giordano S, et al. Adherence to national guidelines for antiemesis prophylaxis in patients undergoing chemotherapy for lung cancer: a population-based study. Cancer 2013;119:1428-1436.

38. Beauchemin M, Sung L, Hershman DL, et al. Guideline concordant care for prevention of acute chemotherapy-induced nausea and vomiting in children, adolescents, and young adults. Support Care Cancer 2020;28:4761-4769.

39. Surbone A, Halpern MT. Unequal cancer survivorship care: addressing cultural and sociodemographic disparities in the clinic. Support Care Cancer 2016;24:4831-4833.

40. Advani PS, Ying J, Theriault R, et al. Ethnic disparities in adherence to breast cancer survivorship surveillance care. Cancer 2014;120:894-900.

41. Mobley EM, Moke DJ, Milam J., et al. AHRQ Comparative Effectiveness Technical Briefs. Disparities and Barriers to Pediatric Cancer Survivorship Care. Rockville, MD: Agency for Healthcare Research and Quality; 2021

42. Castellino SM, Parsons SK, Kelly KM. Closing the survivorship gap in children and adolescents with Hodgkin lymphoma. Br J Haematol 2019;187:573-587

43. Faulk KE, Anderson-Mellies A, Cockburn M, et al. Assessment of enrollment characteristics for Children's Oncology Group (COG) upfront therapeutic clinical trials 2004-2015. PLoS One 2020;15:e0230824.

44. Keegan THM, Parsons HM. Adolescent angst: enrollment on clinical trials Hematology (Am Soc Hematol Educ Program) 2018;2018:154-160. 